\title{
The knowledge, attitudes and beliefs of carers (parents, guardians, healthcare practitioners, crèche workers) around fever and febrile illness in children aged 5 years and under: protocol for a qualitative systematic review
}

Maria Kelly ${ }^{1}$, Laura J Sahm ${ }^{1,2}$, Frances Shiely ${ }^{3}$, Ronan O'Sullivan ${ }^{4,5}$, Maria Brenner ${ }^{6}$, Philip Larkin ${ }^{6}$ and Suzanne McCarthy ${ }^{1,7^{*}}$

\begin{abstract}
Background: Many parents consider fever a disease in itself and feel disempowered when their child is ill. Numerous guidelines have been produced; however, their target audience remains healthcare professionals and not carers of children in general. A reliable source of information will decrease worry in parents and carers when managing a febrile child.

Methods/Design: A systematic search will be conducted in nine electronic databases. Articles published in English, or with an abstract published in English, will be eligible for inclusion in the review. Unpublished literature, grey literature and consultation with experts in the area will be used to supplement database searching. Titles and abstracts of studies will be screened for inclusion in the study by two independent reviewers against pre-determined inclusion and exclusion criteria. A data extraction form will be designed and data will be extracted to provide detail of the included studies by a further two reviewers. Quality assessment of studies will be conducted by two additional independent reviewers and results will be used to moderate included studies. All disagreements will be resolved through discussion until consensus is reached. Thematic synthesis will be used to analyse results.

Discussion: Correct management of fever in children is not well understood in the general population. Although carers can identify fever and febrile illness in children, determination of the severity of fever proves challenging. Research is needed to cohere existing evidence and identify knowledge gaps. It is envisaged that results of this review will contribute to the development of trustworthy, accessible guidelines for parents and carers of children with fever or febrile illness.
\end{abstract}

Systematic review registration: PROSPERO CRD42014009812

Keywords: Fever, Febrile illness, Parents, Carers, Children, Knowledge

\footnotetext{
*Correspondence: S.McCarthy@ucc.ie

${ }^{1}$ Pharmaceutical Care Research Group, School of Pharmacy, University College Cork (UCC), Cork, Ireland

7 Department of Pharmacy, Cork University Hospital, Cork, Ireland

Full list of author information is available at the end of the article
} 


\section{Background}

\section{Description of the current situation}

Fever and febrile illness are some of the most commonly treated childhood illnesses [1-3]. Despite its prevalence, correct management of febrile illness remains unclear in the general population [4-7].

Parents feel disempowered when their child is sick [8] and many interpret fever as a disease in itself [9]. Parents become concerned [6,10-12] and anxious [13-18] when their child has a fever and find it difficult to interpret the severity of their child's illness [8]. The idea of 'fever phobia' was introduced in 1980 by Schmidt and describes unrealistic parental perceptions of fever [19]. Since then, other research has confirmed that parental perceptions are largely unjustified [17,19-23]. Parental responses to fever lead to over engagement with healthcare practitioners and futile consultations [4,24]. Many parents feel that they are not caring appropriately for their child if they are not treating their child's fever [8]. There are numerous cases of unintentional over- and under-dosing with antipyretics each year [25-28]. Despite decades of education and reassurance, parental beliefs are still grounded in the notion that fever is harmful [29-31]. Febrile convulsions remain the focus of parental and many healthcare practitioners concern, and fever is regarded as the main cause of febrile convulsions [6,17,22,24,32-37].

Misconceptions are also present in healthcare practitioners [37-41]. Fear of fever and febrile illness has been observed in nursing staff, leading to variability in practice [24]. Primary-care physicians have also demonstrated varying levels of knowledge regarding the management and risks of a common health problem $[39,40]$. Numerous physicians have exaggerated concerns about fever [41], similar to parental fever phobia.

The aim of this review is to systematically review the knowledge, attitudes and beliefs of carers of children with regard to the management of fever and febrile illness in children.

\section{Why is it important to do this review?}

Currently no systematic review exists on this topic. However, it has been established that research in this area is required [42]. There are relatively few guidelines on this topic [2,42-44], while some of these guidelines suggest that pertinent research is required in the area [42]. The guidelines that are in existence are primarily aimed at healthcare practitioners and neglect many other carers of children including parents [42]. Despite this, there is evidence that there are misconceptions regarding treatment of febrile illness within the healthcare professions [37-41]. Furthermore, studies suggest that there is a lack of understanding of guidelines by carers of children from non-healthcare backgrounds [26-28]. This is supported by misinformation which carers have provided in previous studies [26-28], but to date, carers access to, and understanding of, guidelines is limited. Evidence-based information is required on this topic [45]. This review will contribute to identification of knowledge gaps upon which further research can be conducted by cohering available information on the topic and will be used in the development of guidelines.

This work has utilised stakeholder and knowledge user involvement from its inception. This ensures that the focus of this review and subsequent guidelines address a pertinent research question and knowledge gap for all involved. Ultimately, the results of the review will be beneficial to carers of children, health service personnel and children in general.

\section{Objectives}

To determine the knowledge, attitudes and beliefs of carers around fever and febrile illness in children aged 5 years and under.

\section{Methods/design}

A multidisciplinary review team will be involved in the review. Expertise from emergency department (ED) clinicians and pharmacists (working in both practice and research settings), nurses, systematic review experts and a research design specialist will be obtained. The review will adhere to the criteria outlined below.

\section{Criteria for considering studies for this review Types of studies}

- Original qualitative research;

- Stand-alone qualitative studies;

- Discrete qualitative studies that form part of a larger mixed method study.

\section{Types of clinical setting}

- Hospital emergency departments;

- Other hospital inpatient and outpatient settings;

- General practice surgeries/clinics;

- Childcare facilities;

- Pharmacies;

- Domestic/informal care settings.

\section{Types of participants}

Parents and lay and professional carers of children will be included.

\section{Types of outcome measures}

Qualitative research which explores the knowledge, attitudes and beliefs of parents and lay and professional carers of children ( 5 years of age and younger) with regard to fever and febrile illness in children. 


\section{Search methods for identification of studies}

Published scientific literature will be identified by conducting a systematic search in the following databases:

- CINAHL (EBSCOhost) (from inception to present);

- Cochrane Central Register of Controlled Trials (from inception to present);

- Embase (from inception to present);

- Google Scholar;

- Index to Theses;

- PsycINFO (from inception to present);

- PubMed (from inception to present);

- Turning Research Into Practice (Trip) database (from inception to present); and

- Web of Science (from inception to present).

A reference librarian will be consulted with regard to the design of the search strategy. It is expected that there will be four blocks of terms referring to antipyretic, children, fever and knowledge, involved in the search (Appendix 1). It is envisaged that no qualitative filter will be used. Relevant guidance will be sought from the Cochrane Handbook for Reviews of Interventions [46].

\section{Searching other resources}

Database searching can reveal as low as $30 \%$ of relevant results [47] and qualitative literature can often be found in grey and other literature [48]. Therefore, other methods including the 'pearl growing' method will be used to identify other relevant studies [49]. The following data sources will also be used to identify additional prospective studies:

- Proceedings from scientific meetings;

- Grey literature (theses, internal reports, non-peer reviewed journals) using the OpenGrey database;

- Other unpublished sources;

- Bibliographies of included studies.

Experts and a wide network of contacts will be used to discover other appropriate resources. Therefore our search will be purposive and iterative.

\section{Data collection and analysis Selection of studies}

Titles and abstracts will be screened by two independent reviewers (MK and ROS) in accordance with the PRESS initiative [50]. Should disagreement arise, a third party (LS) will be used to resolve the divergence and reach consensus. All articles which meet the inclusion criteria of the review will be obtained in full text format for further assessment. Two independent reviewers (MK and ROS) will perform second screening of full text journals. All inclusion and exclusion criteria will be applied at this stage.

\section{Data extraction and management}

The specific characteristics of this review, along with other similar reviews will be taken into consideration when designing a data extraction form. Piloting of the data extraction form will take place on a suitable set of reports before it will be used to extract data for this review. Data to provide relevant information about the included studies will be selected from the articles by two independent reviewers (MB and LS). Should disagreement occur, it will be resolved through discussion until consensus is reached.

A comprehensive qualitative data extraction form will be designed based on the specific characteristics of this review and taking other similar reviews into consideration. It will be tested on a suitable set of reports before full application to this study. Two independent reviewers (MB and LS) will conduct the review of relevant articles to extract pertinent details identified in the form. Disagreement will be resolved by discussion until consensus is reached. All information will be stored in a database (QSR International's NVivo software [51]).

\section{Assessment of quality of studies}

The credibility, transferability, dependability and confirmability of each study will be assessed using the Critical Appraisal Skills Programme (CASP) checklist for qualitative studies [52]. This appraisal tool was chosen as:

- It allows rapid evaluation as it has a 10-item checklist; and

- It can be applied in different types of qualitative designs.

Two reviewers (FS and PL) will independently assess the quality of the study. Discussion will be used to resolve any discrepancies. Discrepancies will be referred to a third party should it be required. Study quality will also be examined in the context of the other papers included in the review. Quality assessment will be used to find a balance between the relevance of insights and methodological flaws as methodologically weak studies may offer new insights that may not be present in methodologically strong studies $[53,54]$. No study will be excluded based on quality assessment due to the potential risk of eliminating a valuable insight from a methodologically weak study in the synthesis [53]. Studies which are poorly reported or methodologically weak may offer important new insights grounded in the data [53].

\section{Sensitivity analysis}

Following completion of the synthesis, the results will be analysed to examine which concepts have been derived from which papers. The development of concepts will be linked to the original papers in light of their quality assessment. 


\section{Data synthesis}

Qualitative data will be synthesised using thematic synthesis [55]. Free line-by-line coding of the result sections of included studies will be conducted. Result sections will be obtained from the data extraction forms and will be uploaded into QSR International's NVivo Software version 10 [51] for analysis. Descriptive themes will be developed from the initial codes. The descriptive themes will be used to create analytical themes.

\section{Data analysis}

Preliminary data analysis will be performed by MK. Development of analytical themes will be performed by MK, MB, PL, SMC, ROS, LS and FS in a group session. A table of results detailing the following will be included:

- Bibliographic information;

- Study characteristics;

- Participant characteristics;

- Main findings.

\section{Interpretation of results}

The results will be presented according to analytical themes. The strengths and weaknesses of each study will be discussed. Future research areas will also be debated. The two methods suggested by Popay et al. for assessment of findings will be used in this review [56]:

1. Critical reflection: all correspondence, meetings regarding decisions taken and the rationale for these decisions at each stage of the process will be documented.

2. Input from knowledge users: we will seek input at every stage of the review from knowledge users so that the outcome of the review will be useful.

\section{Discussion}

Carer competence can be influenced by the knowledge, attitudes and beliefs of carers regarding fever and febrile illness. To date, existing research on this topic has not been synthesised. This review aims to address this gap in the literature and to provide further information on the understanding and knowledge of carers regarding fever in children. The review will also serve as a precursor to guideline development. These guidelines will help carers when caring for a child with fever and reduce concerns and anxiety associated with the task.

\section{Appendix 1 Search strategy}

\section{Search terms}

1. Antipyretic agent or analgesic agent or analgesi*.

2. Child or paed* or pedia*.

3. Fever* or febrile* or temperature.
4. Knowledge or attitude* or belief* or view* or opinion* or perception* or concern*.

\section{Abbreviations}

CASP: Critical Appraisal Skills Programme; CRF-C: Clinical Research Facility, Cork; ED: emergency department; Trip: Turning Research Into Practice.

\section{Competing interests}

The authors declare that they have no competing interests.

\section{Authors' contributions}

MK, MB, PL, SMC, ROS, LS and FS conceptualised the study, designed the study protocol and drafted the protocol. MK and ROS will contribute to screening of identified studies for inclusion in the review and final data analysis. MB and LS will extract data from the included studies and will be involved in final data analysis. PL and FS will assess the quality of studies and will be involved in final data analysis. SMC will be involved in final data analysis. MK will draft the final review while MB, PL, SMC, ROS, LS and FS will be involved in editing the final manuscript. All authors will read and approve the final manuscript.

\section{Acknowledgements}

MK is an Honorary Research Fellow at the HRB Clinical Research Facility, Cork (CRF-C). We would like to acknowledge the assistance of Professor Joe Eustace, Director CRF-C, who supplements training and publication costs for this study.

\section{Author details}

${ }^{1}$ Pharmaceutical Care Research Group, School of Pharmacy, University College Cork (UCC), Cork, Ireland. Department of Pharmacy, Mercy University Hospital, Cork, Ireland. ${ }^{3} \mathrm{HRB}$ Clinical Research Facility \& Department of Epidemiology and Public Health, University College Cork, Cork, Ireland. ${ }^{4}$ School of Medicine, University College Cork, Cork, Ireland. ${ }^{5}$ National Children's Research Centre, Dublin 12, Ireland. ${ }^{6}$ School of Nursing, Midwifery and Health Systems, University College Dublin, Dublin, Ireland.

${ }^{7}$ Department of Pharmacy, Cork University Hospital, Cork, Ireland.

Received: 23 October 2014 Accepted: 26 February 2015

Published online: 14 March 2015

\section{References}

1. Eldalo AS. Saudi parent's attitude and practice about self-medicating their children. Arch Pharm Pract. 2013;4(2):57-62.

2. Feverish illness in children: Assessment and initial management in children younger than 5 years. National Institute for Health and Clinical Excellence Clinical Guidelines. 2013.

3. Sullivan JE, Farrar HC, Frattarelli DAC, Galinkin JL, Green TP, Hegenbarth MA, et al. Clinical report - fever and antipyretic use in children. Pediatrics. 2011;127(3):580-7.

4. Walsh A, Edwards H. Management of childhood fever by parents: literature review. J Adv Nurs. 2006:54(2):217-27.

5. Crocetti M, Sabath B, Cranmer L, Gubser S, Dooley D. Knowledge and management of fever among Latino parents. Clin Pediatr. 2009;48(2):183-9.

6. Gupta MS, Rajput U. Parental knowledge, attitude and practices regarding fever in their children: a hospital-based prospective study. Australasian Medical J. 2012;5(1):106.

7. Linder N, Sirota L, Snapir A, Eisen I, Davidovitch N, Kaplan G, et al. Parental knowledge of the treatment of fever in children. Isr Med Assoc J. 1999;1(3):158-60.

8. Kai J. Parents' difficulties and information needs in coping with acute illness in preschool children: a qualitative study. BMJ. 1996;313(7063):987-90.

9. Singhi S, Padmini P, Sood V. Urban parents' understanding of fever in children its dangers and treatment practices. Indian Pediatr. 1991;28(5):501-5.

10. Cinar ND, Altun I, Altınkaynak S, Walsh A. Turkish parents' management of childhood fever: a cross-sectional survey using the PFMS-TR. Australas Emerg Nurs J. 2014;17(1):3-10.

11. Cuzzolin L, Zaffani S, Gangemi M, Elli P, Cremonese P, Chiamenti G, et al. Parental attitudes about the most common symptoms/pathologies in pre-school children. Ital J Pediatr. 2004;30(4):248-53. 
12. Enarson MC, Ali S, Vandermeer B, Wright RB, Klassen TP, Spiers JA. Beliefs and expectations of Canadian parents who bring febrile children for medical care. Pediatrics. 2012;130(4):e905-12

13. Poirier MP, Collins EP, McGuire E. Fever phobia: a survey of caregivers of children seen in a pediatric emergency department. Clin Pediatr. 2010;49(6):530-4.

14. Betz MG, Grunfeld AF. 'Fever phobia' in the emergency department: a survey of children's caregivers. Eur J Emerg Med. 2006;13(3):129-33.

15. Al-Mohsen AHKE. Fever in children: parental perceptions. J Bahrain Medical Society. 2005;17(2):100-6.

16. Al-Nouri L, Basheer K. Mothers' perceptions of fever in children. J Trop Pediatr. 2006;52(2):113-6.

17. Arica SG, Arica V, Onur H, Gulbayzar S, Dag H, Obut O. Knowledge, attitude and response of mothers about fever in their children. Emerg Med J. 2012;29(12):e4.

18. Kramer MS, Naimark L, Leduc DG. Parental fever phobia and its correlates. Pediatrics. 1985;75(6):1110-3.

19. Schmitt BD. Fever phobia: misconceptions of parents about fevers. Am J Dis Child. 1980;134(2):176-81.

20. Andersen AR. Parental perception and management of school-age children's fevers. Nurse Pract. 1988;13(5):8-9. 5

21. Esenay FI, Isler A, Kurugol Z, Conk Z, Koturoglu G. Mothers' approach to feverish child and fever phobia. Turk Pediatri Arsivi. 2007;42(2):57-60.

22. Figueroa FN, Forero J, León JA, Londoño AC, Echandía CA. Detección, Manejo Y Percepción Materna de la fiebre en niños cali - Colombia. Revista Facultad de Medicina de la Universidad Nacional de Colombia. 2012;60(1):40-9.

23. Kwak YH, Kim DK, Jang HY, Kim JJ, Ryu J-M, Oh SB, et al. Fever phobia in Korean caregivers and its clinical implications. J Korean Med Sci. 2013;28(11):1639-44

24. Edwards H, Walsh A, Courtney M, Monaghan S, Wilson J, Young J. Improving paediatric nurses' knowledge and attitudes in childhood fever management. J Adv Nurs. 2007;57(3):257-69.

25. McErlean MA, Bartfield JM, Kennedy DA, Gilman EA, Stram RL, Raccio-Robak N. Home antipyretic use in children brought to the emergency department. Pediatr Emerg Care. 2001;17(4):249-51.

26. Goldman RD, Scolnik D. Underdosing of acetaminophen by parents and emergency department utilization. Pediatr Emerg Care. 2004;20(2):89-93.

27. Purssell E. Parental fever phobia and its evolutionary correlates. J Clin Nurs. 2009;18(2):210-8

28. Boivin JM, Weber F, Fay R, Monin P. Management of paediatric fever: is parents' skill appropriate? Arch Pediatr. 2007;14(4):322-9.

29. Crocetti M, Moghbeli N, Serwint J. Fever phobia revisited: Have parental misconceptions about fever changed in 20 years? Pediatrics. 2001;107 (6):1241-6.

30. Blumenthal I. What parents think of fever. Fam Pract. 1998;15(6):513-8.

31. Betz MG, Grunfeld AF. 'Fever phobia' in the emergency department: a survey of children's caregivers. Eur J Emerg Med. 2006;13(3):129-33. 10.1097/01.mej.0000194401.15335.c7.

32. Karwowska A, Nijssen-Jordan C, Johnson D, Davies HD. Parental and health care provider understanding of childhood fever: a Canadian perspective. CJEM. 2002;4(6):394-400.

33. Polat M, Kara S, Tezer H, Tapisiz A, Derinoz O, Dolgun A. A current analysis of caregivers' approaches to fever and antipyretic usage. J Infect Dev Ctries. 2014;8(3):365-71

34. Sarrell M, Cohen HA, Kahan E. Physicians', nurses', and parents' attitudes to and knowledge about fever in early childhood. Patient Educ Couns. 2002;46(1):61-5.

35. Al-Abdel Jalil HK, Jumah NA, Al-Baghli AA. Mothers' knowledge, fears and self-management of fever: a cross-sectional study from the capital governorate in Kuwait. Kuwait Medical J. 2007;39(4):349-54.

36. Al-Eissa YA, Al-Sanie AM, Al-Alola SA, Al-Shaalan MA, Ghazal SS, Al-Harbi AH, et al. Parental perceptions of fever in children. Ann Saudi Med. 2000;20(3-4):202-5.

37. Poirier MP, Davis PH, Gonzalez-Del Rey JA, Monroe KW. Pediatric emergency department nurses' perspectives on fever in children. Pediatr Emerg Care. 2000;16(1):9-12.

38. Greensmith L. Nurses' knowledge of and attitudes towards fever and fever management in one Irish children's hospital. J Child Health Care. 2013;17(3):305-16

39. Demir F, Sekreter O. Knowledge, attitudes and misconceptions of primary care physicians regarding fever in children: A cross sectional study. Italian J Pediatr. 2012;38(1).
40. Al-Eissa YA, Al-Zaben AA, Al-Wakeel AS, Al-Alola SA, Al-Shaalan MA, Al-Amir AA, et al. Physician's perceptions of fever in children - facts and myths. Saudi Med J. 2001;22(2):124-8.

41. Ipp M, Jaffe D. Physicians attitudes toward the diagnosis and management of fever in children 3 months to 2 years of age. Clin Pediatr. 1993;32(2):66-70.

42. Fields E, Chard J, Murphy MS, Richardson M. Assessment and initial management of feverish illness in children younger than 5 years: summary of updated NICE guidance. BMJ. 2013;346.

43. Caring for children with fever. RCN good practice guidance for nurses working with infants, children and young people. Royal College of Nursing 2008.

44. Melbourne TRCH. Fever in children. 2000.

45. Walsh A, Edwards H, Fraser J. Parents' childhood fever management: community survey and instrument development. J Adv Nurs. 2008;63(4):376-88.

46. Noyes J PJ, Pearson A, Hannes K, Booth A. Qualitative research and Cochrane reviews. In: Higgins JPT, Green S (editors). Cochrane Handbook for Systematic Reviews of Interventions Version 501 [updated September 2008] The Cochrane Collaboration, 2008; Chapter 20.

47. Greenhalgh T, Peacock R. Effectiveness and efficiency of search methods in systematic reviews of complex evidence: audit of primary sources. BMJ. 2005:331(7524):1064-5.

48. Tong A, Flemming K, Mclnnes E, Oliver S, Craig J. Enhancing transparency in reporting the synthesis of qualitative research: ENTREQ. BMC Med Res Methodol. 2012;12:181.

49. Ramer SL. Site-ation pearl growing: methods and librarianship history and theory. J Med Libr Assoc. 2005;93(3):397-400.

50. Sampson M, McGowan J, Cogo E, Grimshaw J, Moher D, Lefebvre C. An evidence-based practice guideline for the peer review of electronic search strategies. J Clin Epidemiol. 2009;62(9):944-52.

51. NVivo qualitative data analysis software. QSR International Pty Ltd. Version 10.

52. CASP. Critical Appraisal Skills Programme. 10 questions to help you make sense of qualitative research [2006]. Available from: http://www.casp-uk.net/ (accessed 2 April 2014).

53. Hannes K. Chapter 4: Critical appraisal of qualitative research. In: Noyes J BA, Hannes K, Harden A, Harris J, Lewin S, Lockwood C (editors), Supplementary Guidance for Inclusion of Qualitative Research in Cochrane Systematic Reviews of Interventions. Version 1 (updated August 2011). Cochrane Collaboration Qualitative Methods Group, 2011.

54. Edwards A, Elwyn G, Hood K, Rollnick S. Judging the 'weight of evidence' in systematic reviews: introducing rigour into the qualitative overview stage by assessing Signal and Noise. J Eval Clin Pract. 2000;6(2):177-84.

55. Thomas J, Harden A. Methods for the thematic synthesis of qualitative research in systematic reviews. BMC Med Res Methodol. 2008;8:45

56. Popay JRH, Sowden A, Petticrew M, Arai L, Rodgers M, Britten N, et al. Guidance on the conduct of narrative synthesis in systematic reviews. In: A product of the ESRC Methods Programme. UK: Lancaster Universit; 2006.

\section{Submit your next manuscript to BioMed Central and take full advantage of:}

- Convenient online submission

- Thorough peer review

- No space constraints or color figure charges

- Immediate publication on acceptance

- Inclusion in PubMed, CAS, Scopus and Google Scholar

- Research which is freely available for redistribution

Submit your manuscript at www.biomedcentral.com/submit

C Biomed Central 